\title{
ISTS 2006-0-1-06 \\ NASA UTILIZATION OF THE INTERNATIONAL SPACE STATION AND THE VISION FOR SPACE EXPLORATION
}

\author{
Julie A. Robinson and Donald A. Thomas \\ Office of the ISS Program Scientist, NASA Johnson Space Center \\ 2101 NASA Parkway, Houston, TX 77058 USA \\ (E-mail : julie.a.robinson@nasa.gov)
}

\begin{abstract}
Under U.S. President Bush's Vision for Space Exploration (January 14, 2004), NASA has refocused its utilization plans for the International Space Station (ISS). This use will now focus on: (1) the development of countermeasures that will protect crews from the hazards of the space environment, (2) testing and validating technologies that will meet information and systems needs for future exploration missions.
\end{abstract}

\section{NASA Utilization Plans for ISS}

Prior to the announcement of President Bush's Vision for Space Exploration [1] on January 14, 2004, NASA's utilization plans for the International Space Station (ISS) focused on a diverse multi-disciplinary research program. This included any and all research, from fundamental to applied, from physical to biological science, from academic to commercial. In response to the Exploration Vision, NASA has refocused its utilization plans for ISS to include three areas.

1. Research on astronaut health and the development of countermeasures that will protect our crews from the space environment during long duration voyages.

2. Testing of research and technology developments that will meet information and systems needs for future exploration missions.

3. Developing and validating operational practices and procedures for long-duration space missions.

NASA's utilization plans for ISS must also be viewed in a programmatic context. Over the near

This is a work of the U.S. Government and is not subject to copyright protection in the United States. Foreign copyrights may apply. term, assembly and safe operations remain NASA's foremost objective for ISS and at the same time a replacement for the Space Shuttle must be developed. ISS assembly goals and prepositioning of anticipated spares prior to Shuttle retirement in 2010 will have significant impacts on NASA research opportunities that may limit utilization upmass, crew time, on-orbit stowage, and down mass even for the highest priority research. From an overall program perspective, major ISS utilization activities are being deferred until after assembly complete. Of necessity, completing assembly of ISS and developing the Crew Exploration Vehicle (CEV) to replace the Space Shuttle must have priority. However, key research activities and utilization are still planned during assembly and will allow us to meet the information needs of the Exploration Vision.

\section{Maximizing Utilization during ISS Assembly}

Given these programmatic limitations, NASA has several strategies for maximizing ISS utilization during the assembly phase. To date, 90 U.S. investigations have been completed or are in process. Wise use of hardware and samples already on orbit can increase scientific return.

\subsection{Multipurpose Facilities}

There are currently five EXPRESS (Expedite the Processing of Experiments for Space Station) racks, two Human Research Facility racks, and the Microgravity Sciences Glovebox available on orbit. The next Shuttle flight (ULF1.1/STS-121, scheduled for mid-2006) will include two additional rack-level facilities that add more research capability. The European Modular Cultivation System (EMCS) will allow for a wide variety of plant growth experiments, with capabilities for microgravity and partial gravity studies. The MELFI (Minus Eighty Laboratory 
Freezer for ISS) rack will add a system of four independently controlled dewars with up to $-80^{\circ} \mathrm{C}$ (Figure 1).

By 2007, additional cold stowage and transportation options will be available including MERLIN (Microgravity Experiment Research Locker/Incubator, $+4^{\circ} \mathrm{C}$ to $-15^{\circ} \mathrm{C}$ ) and GLACIER (General Laboratory Active Cryogenic ISS Experiment Refrigerator, to $-180^{\circ} \mathrm{C}$ ). This hardware will increase the ability to collect and store biological samples on orbit and return them home for analysis.

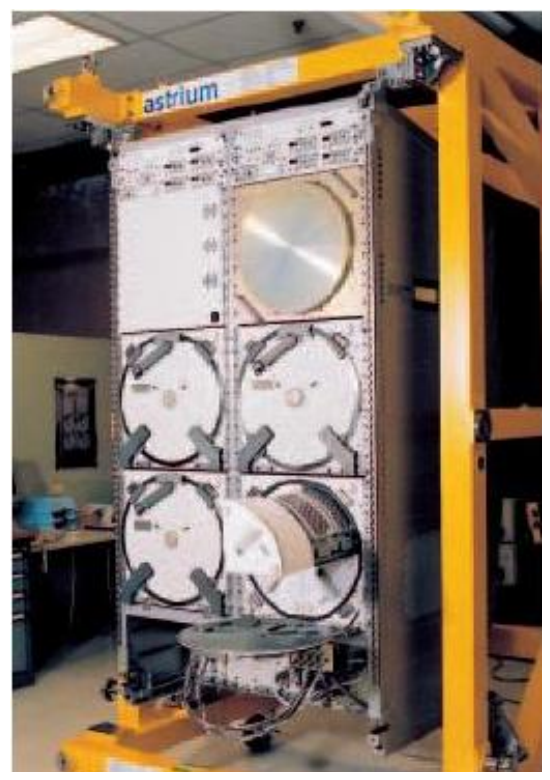

Figure 1. MELFI flight unit. One dewar door is open (bottom right) and a tray partially extracted.

\subsection{Reusable samples}

Another approach to optimizing ISS research is to identify creative proposals for ISS utilization that requires few resources (upmass, crew time, etc.), but still takes advantage of the unique environment in orbit.

An example of reusing samples and finding synergies to get additional research return comes from the Binary Colloidal Alloy Test-3 Critical Point experiment (David Weitz and Peter Lu, Harvard University). This experiment is investigating the growth of colloidal crystals and phase separation effects that can only be studied in space where sedimentation does not occur. To study these effects, frequent photographs need to be taken to show the colloid structure and phase separation as it occurs. During Expeditions 8, 9, and 10 , we relied on the crew to perform the photography and downlink the images. Although the experiment was complete according to the original plan, the results of the data indicated that more frequent imaging of the separation could provide significant new insights into the physical behavior of fluids at the critical point. The samples could be used again for repeat runs, but with limited crewtime, it would be difficult to schedule additional runs. The student Earth observation experiment called EarthKAM (Earth Knowledge Acquired by Middle Schools) cooperated to provide their automated camera system for use by BCAT. During Expedition 12 the EarthKAM hardware was used to perform the image collection function previously accomplished by the ISS crew and also automatically queue the data for near real-time downlink. Images were automatically taken once every two hours for 3-week runs. The automated image gathering and file transfer capabilities of EarthKAM allowed the images to be taken onschedule, regardless of crew availability. Significantly more data will be gathered and with much less crew time. BCAT-2 operations will continue during Expedition 13.

Additional runs of previously completed experiments, use of reusable samples, and testing of new technologies all can help to increase the utilization value of ISS.

\subsection{International Partnerships}

As the ISS is an international endeavor, collaborations with the ISS International Partner space agencies are critical for maximizing research return. In the tradition of the Grenada Crystallization Facility (GCF), international cooperation helps to reduce duplication of facilities, and insure data returns. GCF featured samples prepared by international scientists (led by Hiroaki Tanaka, Japan Aerospace Exploration Agency), in hardware built in Spain for the European Space Agency (ESA), taken to orbit on a Russian vehicle, transferred on the ISS into U.S. hardware (the Commercial Generic Bioprocessing Apparatus, CGBA, to provide thermal conditioning), and later returned on a Russian vehicle [2].

During the recent Expedition 12 on ISS, PromISS-4 (Protein Crystal Growth Monitoring 
by Digital Holographic Microscope, Ingrid Zegers, Vrije Universiteit Brussels, and Belgium) was successfully completed following extensive international cooperation. The objectives of this experiment were to gather data relevant to the depletion model of protein crystal growth. The samples arrived on ISS in a Russian Kubik incubator aboard the Russian Progress 20P vehicle. The hardware, previously used in the Russian segment, was scheduled for transfer to the U.S. Microgravity Sciences Glovebox. U.S. commander and science officer, Bill McArthur, spent extensive discretionary time to locate and assemble the equipment in MSG, saving much of the science. The grown crystals were recently returned with the crew on Soyuz 11S. Analysis of images acquired while the proteins were crystallizing on orbit indicated that depletion zones were observed, and analysis will continue now that samples are on the ground.

New partnerships among International Partners can also help to increase the results as data and hardware are shared among investigators, and help in recovery when there are operational or technical challenges in completing the research. NASA continues to work with all its partners to find areas where research return can be increased.

\section{Planning for the ISS Medical Project}

A final and critical strategy is to focus on getting suitable human physiological data on crewmembers, so that information from each human "subject" living on orbit can be used to better understand the effects of spaceflight on human physiology. Biological sample collection and return requires relatively little upmass and downmass, but has high potential payoff of information for planning future exploration missions.

A complete programmatic review of many aspects of the human research program has been initiated. This review has examined all areas of space clinical physiology, including:

\author{
Neurology and Neurosciences \\ Pharmacotherapeutics \\ Immune Function and Infectious Diseases \\ Nutrition \\ Muscle and Bone Loss \\ Cardiovascular System \\ Behavior and Performance
}

The objective is to join research teams with the NASA flight surgeons that currently maintain the health of the crew. The evidence base for space effects on human health is being combined with the clinical implications to the mission and health of the crew. Finally, gaps in the existing research portfolio are being identified and prioritized so that the most important research for future exploration will be accomplished.

Over the very near term, several gaps have been identified and will be addressed immediately. Studies of the stability of medicines and nutrients in the space environment are being initiated with the next Space Shuttle Flight (STS121/ULF1.1). The newly arrive refrigerated centrifuge will also be used to initiate studies of the changes in key parameters in blood serum during the course of a crewmember's stay on ISS, beginning with the Expedition 14 crew.

\section{Examples of Research Completed on ISS}

In spite of the many challenges to utilizing ISS during the assembly phase, there have been significant research accomplishments that are important for achieving the Exploration Vision. As of the end of March 2006, a total of 78 U.S. investigations have been completed on ISS with an additional 12 investigations ongoing. We will review a selection of these experiments and results, as well as outline some of the ongoing and upcoming research.

Several key experiments on the effects of the space environment on bone loss, and the effectiveness of countermeasures to bone loss have recently been completed. In addition the first study of behavioral health and performance on the International Space Station has been completed, providing insights into the working of crew and ground personnel.

4.1 Sub-regional Assessment of Bone Loss in the Axial Skeleton in Long-Term Spaceflight (Sub-regional Bone), Thomas F. Lang, University of California, San Francisco.

Subregional Bone determined the distribution of bone loss in the spine and hip in long-duration spaceflight using quantitative computed tomography (QCT) and assessed how bone is recovered after return to Earth. One of the first bioastronautics research investigations to begin on ISS, this study recruited 14 subjects from 
Expedition 2 through Expedition 6. All subjects have been home long enough for investigators to have also measured their bone density one year post flight to assess recovery, and these results are expected to be published soon. On ISS, bone mineral density was lost at an average rate of about $0.9 \%$ per month in the lumbar spine and $1.4 \%$ per month in the femoral neck [3]. For comparison, a post-menopausal woman experiences losses of bone mineral on the order of $1 \%$ per year (e.g. [4]). The experiment provides insight into the process of bone loss because it is the first study to differentiate the loss in the cortical bone (the outer part of the bone) and the trabecular bone (the inner parts of the bone). For example, in the hip, losses of mass in the cortical bone averaged around 0.5\%/month whereas losses in the trabecular bone averaged 2.5\%/month [3]. Post-flight measurements of bone recovery have been completed and are now submitted for publications.

These results were from crewmembers who were participating in typical U.S. spaceflight exercise regimens; albeit with exercise routines for some subjects compromised by hardware failures [5]. Now that we better understand the distribution of bone loss, development of countermeasures to control these regional losses is paramount to assure successful human exploration. If Mars mission design includes a zero gravity transit, these countermeasures will be critical to insure that crewmembers are physically capable of completing tasks on Mars and making a safe return. Should an artificial gravity mission design be selected for Mars transit, this understanding of bone loss forms the foundation for gravity prescriptions during transit.

\subsection{Foot/Ground Reaction Forces during Space Flight (Foot), P. Cavanaugh, the Cleveland Clinic.}

The Foot experiment used a biomechanical approach to measuring mechanical loads on the lower extremities. Foot characterized the load placed on legs and feet during daily activities on the ISS. Each participating crewmember was instrumented with sensors-a calibrated forcesensing shoe insole, joint sensors that record angles at the ankle, knee, and hip, and electrodes to record muscle activity in leg muscles. Once actual on-orbit loads are understood, more efficient and focused countermeasures to bone and muscle loss (such as better exercise regimens or equipment) can be developed for exploration missions. The experiment was conducted on four subjects during Expeditions 6, 8, 11, and 12. During Expeditions 11 and 12, special exercise sessions were completed to measure the forces experienced with various settings of the treadmill with vibration isolation system (TVIS), cycle ergometer with vibration isolation (CEVIS), and resistive exercise device (RED).

Preliminary results have already given us great insight into why bone is being lost by crewmembers during their stay on ISS in spite of the exercise protocols in place. Peak forces
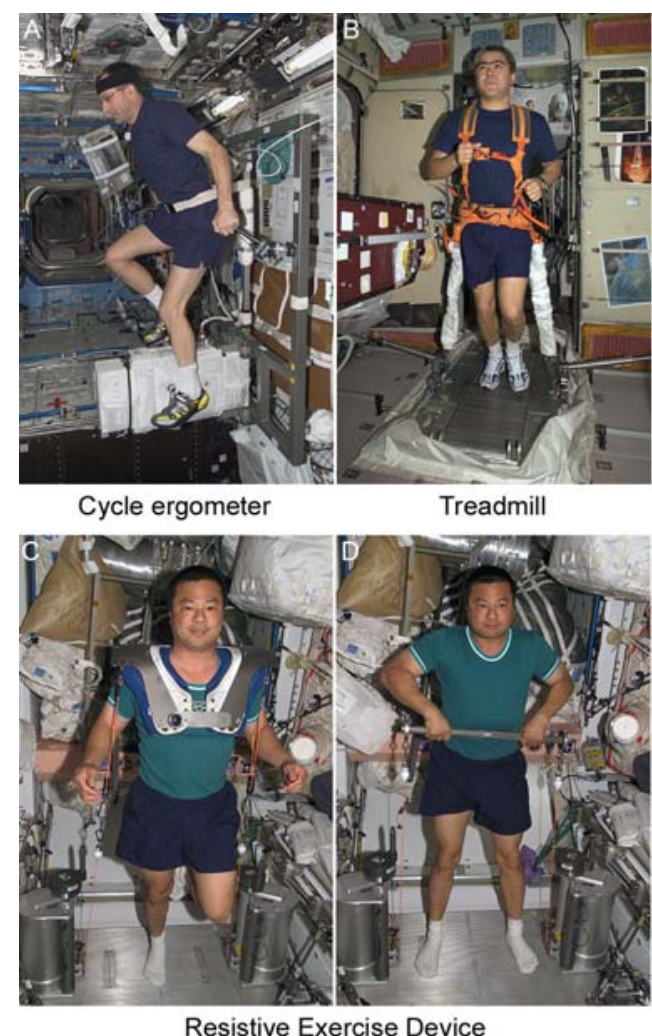

Figure 2. Exercise modalities on ISS. A. Astronaut Donald R. Pettit uses the Cycle Ergometer with Vibration Isolation System (CEVIS) in the Destiny laboratory, ISS006-E13965, 2 January 2003. B. Cosmonaut Salizhan S. Sharipov, equipped with a bungee harness, exercises on the Treadmill Vibration Isolation System (TVIS) in the Zvezda Service Module, ISS010-E-05609, 31 October 2004. C. Astronaut Leroy Chiao, wearing squat harness pads, exercises using the Resistive Exercise Device (RED) equipment in the Unity node, ISS010-E-05325, 28 October 2004. D. Chiao uses the short bar on the SchRED to perform upper body strengthening pull-ups, ISS010-E05343, 28 October 2004. 
experienced during treadmill runs were approximately $63 \%$ of the forces that would have been experienced running on a treadmill on Earth. During 161 days in orbit, bone was lost at a rate of $0.72 \%$ and $2.31 \%$ per month in the total hip and lumbar spine regions, respectively [6], and similar to bone loss documented by the Subregional Bone experiment completed earlier on ISS [3]. Complete results of the exercise trials are expected to be released shortly. These results and recommendations will be important not only for determining proper exercise prescriptions for future crewmembers on ISS, but also in the design and development of exercise countermeasures for exploration missions.

\subsection{Crewmember and Crew-Ground Interaction during International Space Station Missions (Interactions), Nick A. Kanas, Veterans' Affairs Medical Center/University of California-San Francisco, San Francisco, CA.}

Completed during Expeditions 2-9, the Interactions experiment measured the impact of cultural and language background on space missions and evaluated changes over time in interpersonal factors such as tension, cohesion, leadership roles, and the relationship between space crews and monitoring personnel on Earth. ISS crewmembers and mission control personnel responded to questions from three standard mood and interpersonal group climate questionnaires and maintained critical incident logs. Previous studies of crew interactions when U.S. crewmembers were added to Mir crews, identified important patterns of responses in interactions between and among crews and ground personnel (e.g., [7,8]), and many of the behavioral factors studied in this experiment (communication styles, multicultural teams, operational systems) are important in planning operations systems and relationships between exploration crews and ground personnel for lunar and Mars missions.

In contrast to studies of isolated explorers in the Antarctic, preliminary results of this study indicate no change in well-being during the thirdquarter or second-half of a mission [9]. As observed for U.S. crewmembers on Mir, there was evidence of displacement of negative feelings from the crew to the ground, and from ground personnel to outside supervisors [9]. Not surprisingly, the investigation is also identifying differences in mood and group perceptions between Americans and Russians [10] and between crewmembers and mission control personnel [11]. International Space Station (ISS) crewmembers show evidence of improvement in mental health as they adjust to the environment (adaptation), and post-mission surveys of crewmembers are being used to evaluate strategies to enhance crewmembers' in-flight stress tolerance and post-flight adjustment [12].

\section{Conclusion}

The ISS will probably represent the only opportunity to perform on-orbit long-duration studies of human health and performance and technologies relevant for future long-duration missions planned during the next 25 years. It is also our prime opportunity to test systems readiness and operational practices for long duration space missions. Optimal use of ISS will require careful identification of (1) the risks to human explorers and the technological risks for spacecraft systems, (2) the research needed to find ways to reduce these risks, and (3) what research can best be performed on the ISS. Even as NASA focuses on developing the CEV and returning to the moon (2015-2020), making sure that the right experiments are completed on ISS is fundamental to the success of NASA's Exploration Vision.

\section{References}

[1] National Aeronautics and Space Administration, The Vision for Space Exploration, NP-2004-01-334-HQ, NASA Headquarters, Washington, DC, February 2004.

[2]Gindl, H., Scheimann, J., Shirakawa, M., Suvorov, V., and Uri, J.J., "Research on International Space Station - Building a Partnership for the Future," Proceedings of the International Astronautical Congress, IAC-04T.4.04, October 4-8, 2004.

[3]Lang, T., LeBlanc, A., Evans, H., Lu, Y., Gennant, H., and Yu, A., "Cortical and trabecular bone mineral loss from the spine and hip in longduration spaceflight," Journal of Bone and Mineral Research, 19(6), 1006-12, 2004.

[4]Iki, M., Kajita, E., Dohi, Y., Nishino, H., Kusaka, Y., Tsuchida, C., Yamamoto, K., and Ishii, Y., "Age, menopause, bone turnover 
markers and lumbar bone loss in healthy Japanese women,” Maturitas, 25(1), 59-67, 1996.

[5] Rhatigan, J.L., Robinson, J.A., Sawin, C.F., "Exploration - Related Research on ISS: Connecting Science Results to Future Missions," 44th AIAA Aerospace Sciences Meeting and Exhibit, Reno, NV. AIAA 2006-344, 2006 (Also published as a NASA/TP 2005-213166).

[6]Cavanagh, P. R., Maender, C., Rice, A.J., Gene, K.O., Ochia, R.S., and Snedeker, J.G., "Lower-extremity loading during exercise on the International Space Station," Transactions of the Annual Meeting of the Orthopaedic Research Society, No. 0395, 2004.

[7]Kanas, N., Salnitskiy, V., Grund, E.M., Weiss, D.S., Gushin, V., Kozerenko, O., Sled, A. and Marmar, C.R., "Human interactions in space: Results from Shuttle/Mir," Acta Astronautica, 49, 243-260, 2001.

[8]Kanas, N., Salnitskiy, V., Weiss, D.S., Grund, E.M., Gushin, V., Kozerenko, O., Sled, A., Bostrom, A. and Marmar, C.R., "Crewmember and ground personnel interactions over time during Shuttle/Mir space missions," Aviation, Space and Environmental Medicine, 72, 453-461, 2001.

[9] Kanas, N., Salnitskiy, V.P., Ritsher, J.B., Gushin, V.I., Weiss, D.S., Saylor, S., and Marmar, C., "Human interactions in space: ISS versus Shuttle/Mir," Proceedings of the International Astronautical Congress, IAC-05A1.5.02, Oct. 17-21, 2005.

[10] Ritsher, J.B., Kanas, N., Gushin, V.I., and Saylor, S., "Cultural differences in patterns of mood states on board the International Space Station," Proceedings of the International Astronautical Congress, IAC-05-A1.5.03, Oct. 17-21, 2005.

[11] Clement, J. and Ritsher, J.B., "Operating the ISS: Cultural and leadership challenges," Proceedings of the International Astronautical Congress, IAC-05-A1.5.05, Oct. 17-21, 2005.

[12]Ritsher, J.B., Kanas, N., and Ihlea, E.C., "Psychological adaptation and salutogenesis in space: lessons from a series of studies," 15th IAA (International Academy of Astronautics) Humans in Space Symposium, Graz, Austria, May 22-26, 2005. 